\title{
SCIENCES ET PHILOSOPHIE DES LUMIÈRES
}

Materia actuosa. Antiquité, âge classique, Lumières. Mélanges en l'honneur d'Olivier Bloch, recueillis par Miguel Benitez, Antony McKenna, Gianni Paganini et Jean SAlem. Paris, Honoré Champion, 2000. 15,5 × 22,5, 755 p. (Champion-Varia, 38).

Né en 1930, Olivier Bloch a consacré, depuis sa thèse de doctorat d'État (La Philosophie de Gassendi. Nominalisme, matérialisme et métaphysique) en 1970, une infatigable activité de chercheur et d'enseignant à l'étude du matérialisme dans ses différents aspects. Par les livres qu'il a écrits, par les textes qu'il a édités, par les très nombreux articles qu'il a publiés, ainsi que par tous les colloques, rencontres et séminaires qu'il a organisés, il a grandement aidé à une meilleure connaissance des traditions libertines de l'âge classique et de leur prolongement dans le matérialisme des Lumières ; il a contribué à la formation de générations de chercheurs. Les nombreuses contributions de ce recueil, riches et variées, lui rendent un hommage mérité et témoignent bien de la diversité de ses champs de recherche sous l'unité de cette thématique matérialiste. Si la profusion de l'ensemble rend évidemment dérisoire l'idée d'une synthèse qui rendrait justice à chacun des quarante et un articles qui composent ce volume, on peut du moins tenter d'en décrire les grandes lignes.

Sur le plan chronologique, ces études portent sur une période qui va de l'Antiquité au $\mathrm{XX}^{\mathrm{e}}$ siècle, puisque les premiers articles sont consacrés à Homère (Marcel Conche), aux présocratiques (Antonio Negri), à la formule d'Euripide « qui sait si vivre n'est pas mourir et si mourir n'est pas vivre »(Jean Salem) et à Lucrèce (Alain Gigandet), et les derniers s'intéressent à Maurice Merleau-Ponty et Alexandre Kojève (Pierre Macherey), Collingwood (Paulette Carrive) et Anatole France (Jean Deprun). Mais, en réalité, l'essentiel du volume porte sur la période qui va de la Renaissance à la fin du XVIII ${ }^{\mathrm{e}}$ siècle. Dans ce cadre, les auteurs sur lesquels Bloch a travaillé sont bien sûr le sujet d'un certain nombre d'études : Cyrano de Bergerac (Madeleine Alcover, Margaret Sankey), Gassendi (Gianni Paganini, Wallace Kirsop, Rainer Specht) et surtout Spinoza qui, à lui seul, est l'occasion d'un petit recueil interne à ce vaste volume (Pierre-François Moreau, André Tosel, Theo Verbeek, Luisa Simonutti, Miguel Benitez, Laurent Bove).

Les parties consacrées au XVIII ${ }^{\mathrm{e}}$ siècle et à la philosophie clandestine sont peut-être, au sein de ce riche ensemble, les plus nouvelles dans leurs contenus. De nombreuses études viennent en effet mettre en valeur des auteurs peu étudiés, tels Anacharsis Cloots (Roland Mortier), Jean Meslier (Gianluca Mori), Joseph Priestley (Ann Thomson), Jean-Partocle Parisot (Alain Mothu), Balthasar Bekker (Wiep Van Bunge) ou encore Robert Challe (Frédéric Deloffre). Deux textes clandestins appartenant au même recueil manuscrit sont édités par Antony McKenna et Élisabeth Quennehen, auxquels deux études, consacrées à la nature de ces recueils clandestins (Geneviève Artigas-Menant) et aux rapports de Voltaire à cette littérature clandestine (Marie-Hélène Cotoni), apportent un heureux complément.

Il n'est pas possible de tout citer dans ce volume. Il est à l'image de celui auquel il rend hommage, sérieux et généreux, lançant quantité de pistes pour des recherches futures. 
Jeff Loveland, Rhetoric and natural history. Buffon in polemical and literary context. Oxford, Voltaire Foundation, University of Oxford, 2001. 15,5 × 24, X-214 p., bibliogr., index (SVEC, $2001:$ 03).

Autant l'étude de Buffon scientifique et philosophe s'est considérablement développée depuis les années 1950 - compensant ainsi le relatif discrédit dans lequel cet auteur si célébré $\mathrm{au} \mathrm{XIX}{ }^{\mathrm{e}}$ siècle était tombé depuis le début du $\mathrm{XX}^{\mathrm{e}}$ siècle - autant celle de Buffon littéraire, auteur d'une théorie et d'une pratique de l'expression, semble, par contraste, avoir été de manière générale trop négligée.

Le livre de Jeff Loveland entend combler ce manque et met en avant l'importance de la dimension rhétorique pour comprendre l'ensemble du discours de Buffon. La pertinence de son projet consiste plus précisément à ne pas traiter de façon séparée la dimension du style chez cet auteur, comme si c'était là une question extérieure de pure forme. Il s'agit au contraire de l'articuler de façon synthétique au contenu scientifique et philosophique de l'œuvre, de façon à en faire un instrument d'intelligibilité propre à en éclairer de façon nouvelle les apparences d'obscurité, de contradiction, les exagérations et les changements déconcertants de point de vue. Longtemps, selon Loveland, malgré des travaux importants, l'insuffisance de prise en compte de cette dimension rhétorique a empêché de comprendre de façon satisfaisante et complète le discours de Buffon. Au-delà du cas de cet auteur, ce parti pris de lecture nous semble participer d'une attitude qui cherche à ressaisir de façon davantage interne la sensibilité particulière de cette époque et à prendre en charge des aspects souvent spontanément reçus de notre point de vue comme frivoles et superficiels et pourtant si prégnants pour leur redonner toute leur signification. L'importance particulière que cette dimension rhétorique vient prendre chez Buffon est liée par Loveland, d'une part, au souci de plaire aux divers publics que peut toucher l'Histoire naturelle - et de répondre à la préoccupation esthétique omniprésente qui y est conjointe - et, de l'autre, au contexte polémique - avec les positions extrêmes qu'il engendre - dans lequel l'œuvre vient s'insérer. Ainsi, un bénéfice non négligeable de cette perspective est de relativiser et de dédramatiser fortement les contradictions que les points de vue plus traditionnels, davantage centrés sur le contenu théorique du discours, avaient souvent excessivement mises en relief. Loin de se borner à l'œuvre de Buffon, mais la réinsérant au contraire dans le contexte concret de la culture polémique et rhétorique du temps, l'intérêt de ce travail est également de procéder à une véritable mise en situation de tous les problèmes de l'époque, les faisant redécouvrir sous un nouvel angle tout en déployant à chaque fois le panorama synthétique des positions des autres auteurs. Loveland assouplit les notions et les enjeux traditionnellement étudiés : il les réintroduit dans une pratique d'écriture et un jeu de relations expliquant leurs différentes, et souvent extrêmes, modulations sans aller toutefois jusqu'à y absorber leur rigueur conceptuelle.

Très documenté et précis, clair et toujours nuancé, ne tombant jamais dans l'impasse du « tout rhétorique », cet ouvrage montre la fécondité de sa perspective à travers six chapitres qui portent sur autant de problèmes fondamentaux de l'époque : la préoccupation, si chère à Buffon, du style ; le thème, d'une histoire fort chargée, des causes finales ; l'image, bien ancienne également, de la « chaîne des êtres »; le problème du statut, si contesté alors, des théories et des systèmes ; l'emploi des mathématiques (qui aurait pu sembler à tort à l'écart de toute préoccupation rhétorique) ; le nominalisme, enfin, position devenue dominante et plus ou moins obligée.

Les trois premiers ordres de problèmes renvoient davantage, selon les termes de l'auteur, à une « rhétorique » de la "séduction », répondant ainsi à l'augmentation considérable des amateurs de sciences au XVIII ${ }^{\mathrm{e}}$ siècle, tandis que les trois derniers, plus scientifiques, mettent plutôt en œuvre une « rhétorique » proprement polémique dans une communauté savante 
extrêmement déchirée. L'ouvrage montre d'abord l'ambivalence du style chez Buffon, source à la fois de sa force auprès d'un vaste public qui en est particulièrement amateur et de sa faiblesse vis-à-vis des critiques qui ne manquent pas de s'emparer d'une qualité si éminente pour dénoncer les excès de l'imagination et l'attachement à des considérations littéraires plus que scientifiques. C'est aussi, semble-t-il, par souci du goût du public que Buffon, théoriquement si critique des causes finales comme une grande partie des auteurs de son temps, se complaît pourtant constamment à les évoquer à travers les figures de son langage, reproduisant en cela une contradiction - également très commune - entre déclarations doctrinales et méthodologiques d'une part et pratiques d'écriture de l'autre. La notion de chaîne des êtres, quant à elle, est utilisée selon un double jeu plus subtil qui, d'un côté, exploite encore sa popularité et son image d'orthodoxie, et, de l'autre, multiplie les exceptions et les critiques à son égard pour s'accorder à d'autres exigences - comme celle de la différence du vivant et de la matière inerte ou de l'homme et de l'animal - qui font aussi le souci des lecteurs. L'idée de chaîne des êtres, ainsi, sert à Buffon de support pour rendre « aimable » son discours en lui permettant de développer ses « grandes vues » sur la nature, et d'élever son auditoire à des considérations esthétiques et morales supérieures. Elle lui est utile également pour critiquer les découpages arbitraires des taxinomistes ou pour familiariser à l'idée d'une certaine transformation des espèces dans le temps. Mais cette même notion s'avère aussi, à l'égard d'autres perspectives, constituer un obstacle qu'il lui faut régulièrement contourner sans trop marquer la contradiction. Cet usage à profil variable de telles notions clefs - qui joue de leur vague et de leur ambiguïté pour les adapter à des publics différenciés ou aux demandes multiples - se repère également chez les autres auteurs contemporains de Buffon, mais apparaît chez lui particulièrement marqué et contrasté.

Quant aux trois derniers thèmes - la notion de système, le statut des mathématiques, la critique nominaliste des idées générales - l'attitude de Buffon est double encore. Il s'agit pour lui de se camper à leur égard dans une posture officielle de refus intransigeant : les systèmes vont être condamnés sous le motif majeur de leur caractère hypothétique, les mathématiques sous celui de leur abstraction, les idées générales sous celui de leur incapacité à rendre compte d'une réalité concrète toujours individualisée. L'on pourra ainsi en façade utiliser ces instruments de façon polémique à l'encontre des adversaires, mais tout en connaissant bien l'impossibilité par ailleurs d'une telle exclusion dans la pratique effective, compte tenu des exigences méthodologiques d'un travail scientifique - en particulier de classification pour une entreprise comme l'Histoire naturelle - et également de l'ambition propre à Buffon de déployer de vastes synthèses, de surcroît souvent habillées d'une rhétorique mathématique. Tout en insistant sur la dimension stratégique forte du discours savant en général au XVIII ${ }^{\mathrm{e}}$ siècle et singulièrement de celui de Buffon, le regard critique qui fait la perspective de l'ouvrage ne tombe pas pour autant dans le scepticisme en plongeant ce discours dans le simple souci opportuniste, mais marque constamment les limites de sa propre thèse en prétendant seulement compléter et relativiser une approche purement théorique de ce siècle. Loveland rend compte des contradictions - nombreuses et irréductibles sur un plan seulement doctrinal - que l'on recense chez Buffon, dès la parution de son œuvre, en les déplaçant sur un autre plan où elles apparaissent liées à une situation, à la fois exemplaire et poussée à l'extrême chez cet auteur, de déchirement entre les trois exigences divergentes que sont la construction de la vérité scientifique, les attentes très esthétiques et moralisantes d'un public élargi, et celles enfin, fort susceptibles et irritables, des élites savantes.

Pourvu d'un index détaillé des auteurs et d'une bibliographie riche et actualisée, qui manque cependant d'une distinction marquée entre écrivains d'époque et commentateurs modernes, ce livre - dont on peut juste regretter peut-être une certaine répétitivité de la thèse malgré la diversité des domaines abordés - représente une étude subtile et intéressante de 
l'œuvre d'une très grande figure des Lumières. Au-delà se trouvent également éclairés les mécanismes discrets - reliant les pratiques d'écriture, les conflits en apparence seulement purement doctrinaux entre les acteurs, et le jeu de la reconnaissance et du succès - qui soustendent le travail de la réflexion et de la recherche à cette époque remarquablement polémique de notre histoire.

Jean-Luc GuICHET

Pierre-Paul LE MERCIER DE LA RIVIÈRE, L'Ordre naturel et essentiel des sociétés politiques. Texte revu par Francine MARKOviTs. Paris, Fayard, 2001. $14 \times 22,5,543$ p. (Corpus des œuvres de philosophie en langue française).

La réédition de L'Ordre naturel et essentiel des sociétés politiques est une excellente nouvelle pour tous les historiens des idées spécialistes du siècle des Lumières. Le texte a été collationné d'après l'édition de 1910 (par Edgar Depitre) avec une vérification effectuée sur l'édition originale in-douze, en deux volumes, de 1767 (exemplaire de la bibliothèque de l'Institut). L'édition elle-même nous a semblée excellente : la typographie et la mise en page sont agréables et le choix de maintenir l'orthographe originelle est heureux (le compliment peut être étendu à l'ensemble de cette collection). Saluons ici la précision avec laquelle Francine Markovits indique la source du texte de l'édition qu'elle a préparée, cet effort est suffisamment rare pour mériter mention. Toutefois, il faut noter que, comme pour l'Ami des hommes et la Philosophie rurale, L'Ordre essentiel a été publié simultanément en in-quarto et en in-douze : il y a donc non pas une, mais deux éditions originales ! C'est d'ailleurs l'édition in-quarto qui avait servi pour l'édition de 1910. La mention de deux éditions ultérieures datées de 1768 en format in-quarto et in-dix-huit est plus surprenante. Ces deux éditions ne sont pas à la Bibliothèque nationale de France et elles n'apparaissent pas non plus dans les catalogues de livres anciens disponibles sur internet. On peut se demander si Louis-Philippe May, qui est la source de Markovits, n'a pas confondu le livre de Pierre-Paul Le Mercier de La Rivière avec celui de Pierre-Samuel Du Pont de Nemours, De l'origine et des progrès d'une science nouvelle, effectivement paru en 1768, et qui se présente comme un résumé de l'ouvrage de Le Mercier. Il n'aurait pas été le premier d'ailleurs, puisque Adam Smith semble faire la même confusion lorsqu'il évoque le "petit ouvrage » de Le Mercier dans la Richesse des nations. Il est tout de même dommage que dans une si courte notice, on trouve deux informations douteuses qui aurait pu être corrigées avec un peu d'attention. Ces imprécisions sont encore plus criantes dans le dossier publié dans le numéro 40 de la revue Corpus par Markovits, qui est conçu comme une introduction à la lecture de la réédition de L'Ordre naturel et essentiel des sociétés politiques. La présentation générale oublie de mentionner des travaux importants, en particulier ceux de Reinhard Bach sur Le Mercier, Rousseau et Sieyès ou, de manière plus surprenante encore, de Catherine Larrère. Les fiches de personnages importants n'indiquent pas leurs sources, et plusieurs ne sont pas non plus mentionnées dans la bibliographie générale. Enfin, de nombreuses coquilles, en particulier dans la bibliographie générale, contribuent à renforcer l'impression générale d'un certain manque d'attention (G. Steiner pour Philippe Steiner ; le Journal de commerce est confondu avec le Journal du commerce, d'agriculture, et des finances ; J. Egrert pour J. Egret).

Toutefois, ces critiques ne doivent pas conduire à négliger l'importance de la réédition de ce texte dont l'impact sur les contemporains reste largement sous-estimé. Pendant trop longtemps, il n'a guère été parcouru que par les spécialistes de l'école physiocratique et 
mentionné pour son exposition de la théorie du despotisme légal. Or, l'ouvrage a connu un réel succès au moment de sa parution en 1767. Quelques semaines après le départ de Le Mercier en Russie, où sa réputation allait être ternie aux yeux de ses contemporains et de la postérité par les rebuffades et les railleries de Catherine II, Du Pont lui écrit que plus de 5000 exemplaires de son ouvrage se sont écoulés. Il n'y a donc pas que Diderot qui se passionne pour L'Ordre naturel et son auteur (c'est lui qui a incité Catherine II à le faire venir). Le succès de l'ouvrage peut se mesurer au nombre et à la stature de ses critiques. Mably, Linguet s'attaquent à sa théorie politique ; Graslin à sa théorie économique. Enfin, Forbonnais et son cousin Plumard de Dangeul, rescapés du groupe Gournay, le « déchirent », dixit Du Pont, dans les pages du Journal de l'agriculture, du commerce et des finances de 1767 et 1768 .

Il est donc à espérer que cette réédition permette une relecture plus ouverte de ce « classique » de la pensée des Lumières. On notera en effet que plus de la moitié de l'ouvrage (à partir du chapitre XXVII) est consacrée aux questions économiques ; on sait d'ailleurs la part importante qu'a pris Quesnay dans sa rédaction. On y trouve des discussions importantes sur la valeur, la nature du produit net, le commerce international et la stérilité du commerce. Plusieurs point méritent d'être redécouverts. Tout d'abord, Le Mercier reconnaît la nécessité d'un profit pour les activités non-productives (commerce et industrie) car : « le commerçant [...] doit être indemnisé de tous ses frais ; il lui est dû en outre, des salaires et des intérêts pour toutes les sommes qu'il est dans le cas d'avancer» (p. 396). Il développe ce point de manière claire et détaillée à propos de l'exemple de l'industrie de la dentelle : « 1000 écus sont le prix nécessaire de la dentelle; prix nécessaire formé par le montant de toutes les dépenses que les fabricants ont à faire pendant le temps qu'ils emploient à cet ouvrage ; par d'autres dépenses encore de divers ouvriers qui concourent à la préparation des lins ; par celles aussi du marchand qui fait les avances de ces dépenses ; par les intérêts qu'il doit retirer de ces mêmes avances ; par les rétributions dues aux peines qu'il se donne personnellement ; par la valeur des différents risques auxquels son commerce expose» (p. 431).

À lire ces lignes où, non seulement Le Mercier reconnaît la nécessité d'un profit, mais le décompose en catégories analytiques précises - intérêt du capital avancé à la production, rémunération du travail de l'entrepreneur (organisation), rémunération du risque de l'entreprise - auquel s'ajoute le coût de production à proprement parler, qui comprend les salaires et les dépenses en matières premières et outils de production, on a bien du mal à comprendre pourquoi les historiens de l'école physiocratique continuent, à la suite de Marx, à proposer moult interprétations de l'absence d'une théorie du profit chez les physiocrates ! On trouve également dans l'ouvrage de Le Mercier l'idée d'une interdépendance générale des prix des biens dans l'économie réglée par la concurrence : «[...] toutes les valeurs vénales ont entre-elles un équilibre habituel et nécessaire ; le prix des unes décide du prix des autres » (p. 402). Il développera d'ailleurs ce point dans son ouvrage de 1770, L'Intérêt général. Sur tous ces points, si Le Mercier ne se singularise pas par rapport à Quesnay, on doit bien avouer que son langage est bien plus compréhensible.

La théorie politique de Le Mercier mérite également mieux que l'étiquette infamante que lui confère l'expression «despotisme légal». On commence seulement à comprendre aujourd'hui que Le Mercier n'est pas seulement l'auteur d'un livre, mais un auteur qui, dans les années qui séparent la publication de L'Ordre essentiel de la Révolution française, a contribué notablement au débat sur la nature du bon gouvernement. Cette reconsidération a d'ailleurs été entamée dans plusieurs contributions insérées dans le volume des Études Jean-Jacques Rousseau pour l'année 1999. Pour conclure, il faut donc se féliciter de cette 
nouvelle édition et espérer qu'elle incite à la republication de quelques autres des textes politiques importants des disciples de Quesnay.

Loïc CHARLES

Jean-Jacques Rousseau et la chimie. Textes réunis par Bernadette BENSAUDE-VINCENT et Bruno Bernardi, Corpus, 36. Nanterre, Centre d'études d'histoire de la philosophie moderne et contemporaine, 1999. 14,7 ×20,7, $206 \mathrm{p}$.

Ce numéro rassemble une série d'articles sur le rapport de Rousseau à la chimie de son siècle et le rôle de la chimie dans sa pensée sociale, anthropologique et politique. La première partie montre en quoi les Institutions chymiques, traité de chimie rédigé par Rousseau, loin d'être un texte marginal, s'insère à part entière dans l'itinéraire intellectuel et dans l'œuvre de Rousseau. La seconde partie montre en quoi la chimie constitue une référence, un « modèle », voire un « paradigme » (Bruno Bernardi, p. 193-194), pour la pensée rousseauiste en général. Ce volume témoigne d'un intérêt nouveau pour la figure peu connue d'un Rousseau chimiste et pour sa philosophie en tant qu'elle emprunte certains de ses concepts à la chimie. À côté de la musique et de la botanique, il faut « compter la chimie au nombre des univers de référence du citoyen de Genève » (Bernadette Bensaude-Vincent, p. 6). Cet ouvrage souligne plus largement la prépondérance des sciences dans la genèse de la pensée rousseauiste, à côté de l'image traditionnelle d'un Rousseau pourfendeur des sciences. Si les Institutions chymiques ne forment pas une œuvre originale, mais un travail de compilation, de traduction et de réécriture des principales doctrines chimiques de Guillaume François Rouelle, Johann Joachim Beccher, Georg Ernst Stahl, Jean-Baptiste Sénac, Hermann Boerhaave, elles présentent un double intérêt. Du point de vue de l'histoire des sciences, elles révèlent l'état des connaissances de Rousseau sur la chimie de son temps. Du point de vue de l'étude génétique des textes, elles exposent les concepts chimiques dont s'inspire toute l'œuvre rousseauiste.

L'article introductif de Bensaude-Vincent et Bernardi, propédeutique à la lecture des Institutions chymiques, retrace l'évolution des rapports entre Rousseau et la chimie. Rousseau s'intéresse d'abord, dans l'entourage de Madame de Warens, aux pratiques et à l'art des chimistes. Il se familiarise précocement avec les instruments, opérations et termes techniques de l'art spagyrique (ancien nom de la chimie médicale empirique). Puis il parfait sa connaissance de la science chimique, en suivant à Paris, avec son ami Dupin de Francueil à partir de 1753, les cours de chimie de Rouelle, tout comme Diderot, d'Holbach, Condorcet, Voltaire ou encore de futurs chimistes, tels que Lavoisier et Macquer. Enfin, il met ses connaissances en application en effectuant des travaux de laboratoire à Chenonceau entre 1755 et 1757 .

La première partie de l'ouvrage, « Rousseau dans la chimie du XVIII ${ }^{\mathrm{e}}$ siècle », comprend 4 articles. L'article de Bernard Joly porte sur « la question de la nature du feu dans la chimie de la première moitié du XVIII ${ }^{\mathrm{e}}$ siècle » et montre en quoi les Institutions chymiques reflètent les débats sur la nature et les propriétés du feu qui, en France à la fin du XVII et au début du XVIII ${ }^{\mathrm{e}}$ siècle, opposent théories mécanistes substantialistes (Descartes, Malebranche, Lémery), théories dynamistes newtoniennes (Boerhaave, Voltaire) et théories du phlogistique de Stahl (Sénac, Rouelle). La théorie rousseauiste du feu, fondée sur un refus du mécanisme cartésien et de l'attraction newtonienne et synthétisant celles de Boerhaave et de Stahl, témoigne de l'évolution qui s'opère dans la chimie française à la fin des années 1740. 
L'article de Jonathan Simon, «L'homme de verre. Les trois règnes et la promiscuité de la nature », montre en quoi les Institutions chymiques héritent de la théorie des trois règnes (minéral, végétal et animal) du chimiste Beccher et de son idée d'une circulation de la matière entre les règnes au moyen du processus chimique de vitrification. Selon Beccher, il est possible de transformer hommes et animaux en verre. Comme Beccher, Rousseau pense une continuité des règnes, mais contre lui, il refuse l'espoir chimérique et monstrueux d'une forme de vie artificielle. Cet article rappelle l'étroite imbrication de la chimie française du XVIII ${ }^{\mathrm{e}}$ siècle avec l'artisanat des maîtres verriers et le commerce du verre, dont la valeur n'est pas seulement marchande, mais aussi esthétique et spirituelle. Rousseau, reprenant la vision apocalyptique d'un anéantissement du monde par vitrification, participe à cette tradition de valorisation du verre.

L'article de Bensaude-Vincent souligne «l'originalité de Rousseau parmi les élèves de Rouelle ». Quoique Rouelle ne soit pas le chimiste le plus cité des Institutions chymiques, Rousseau est influencé par l'enseignement rouellien, qu'il se réapproprie de manière originale. L'héritage rouellien de Rousseau diffère en effet de celui de Diderot, Venel, d'Holbach, Turgot ou Macquer. Cet article révèle un Rousseau « profondément imprégné de l'enseignement de Rouelle, partageant l'essentiel des idées de ses contemporains sur les concepts de base de la chimie et sur les orientations théoriques, notamment dans la critique du mécanisme » (p. 101). Rousseau compose ainsi son propre univers chimique par une recréation de l'œuvre rouellienne.

L'article de Marco Beretta, «Jean-Jacques Rousseau et la chimie », se propose de reconstruire « la chronologie et le contexte intellectuel des Institutions et de donner une explication plausible des motifs qui conduisirent Rousseau à abandonner complètement la chimie après 1757 » (p. 104). Si, à la fin de sa vie, Rousseau considère la chimie comme une vaine ambition (les Rêveries du promeneur solitaire dénoncent l'aspect coûteux, long, pénible, dangereux et contradictoire des expériences de chimie en laboratoire), il n'en va pas de même au début, où il étudie la chimie en amateur éclairé. Dans les Institutions, il tente de donner à la chimie un statut scientifique et philosophique et propose une « réévaluation épistémologique de la chimie » (p. 115). À l'instar de Rouelle, il veut fonder la légitimité de la chimie en l'arrachant au rôle de simple art auxiliaire de la médecine et en la séparant de l'alchimie. Pour Rousseau, la chimie est un « instrument cognitif qui, à travers une procédure expérimentale assez aisée, permet de suivre le déroulement dynamique des réactions, sans s'engager dans des conjectures métaphysiques » (p. 109). Son intérêt pour la chimie vient de son souci de combattre les systèmes spéculatifs en général et de justifier empiriquement la thèse antimécaniste. La chimie, dont l'objet principal est la décomposition (analyse) des corps en leurs parties constituantes, en montrant qu'il est impossible d'isoler les principes ultimes de la matière, conduit en effet à condamner le mécanisme.

La seconde partie de l'ouvrage, «La chimie dans la pensée de Rousseau », comprend 3 articles. L'article de Florent Guénard, « Convenances et affinités dans la Nouvelle Héloïse », compare les relations hommes-femmes dans la Nouvelle Hélö̈se aux types d'union matérielle dans les Institutions chymiques. Rousseau distingue l'union fondée sur la reconnaissance réciproque d'une identité de caractères (les amants, Saint-Preux et Julie, se conviennent parce qu'ils se ressemblent) et l'union de complémentarité entre deux êtres hétérogènes (les époux, Wolmar et Julie, se conviennent parce qu'ils se complètent). Ces deux types d'union correspondent à deux types d'affinités chimiques. L'union entre amants relève d'une affinité par homogénéité, d'une attirance par similitude (des âges, des goûts, des affections, des pensées). L'union entre époux, seule durable, relève d'une affinité par hétérogénéité.

L'article de Martin Rueff, «L'élément et le principe. Rousseau et l'analyse », montre que l'anthropologie rousseauiste ne s'appuie ni sur la méthode géométrique, ni sur la causalité 
mécanique cartésienne, mais sur la science chimique qui lui fournit un critère et une méthode fondés sur l'expérience. Le but de cette anthropologie étant de connaître l'homme naturel, il lui faut séparer, selon un protocole expérimental emprunté à la chimie, l'artificiel de l'originel. «Lire les Institutions chymiques, c'est se demander ce que Rousseau retient de la chimie pour construire sa théorie de l'homme » (p. 144). La chimie fournit à Rousseau un moyen de comprendre la nature humaine et de critiquer un certain concept de l'élément et de l'analyse. Rousseau rejette toute conception du simple comme atome absolu. Les Institutions éclairent négativement sa méthode anthropologique. «La chimie n'est pas tant le modèle des opérations du philosophe que l'indice d'un embarras de méthode : l'analyse ne parvient pas à remonter au simple, au principe » (p. 147). À une ontologie atomiste du lien social, Rousseau préfère une anthropologie holiste, où le simple n'est pas tant point de départ objectif et absolu pour la constitution des corps que point d'arrivée subjectif, provisoire et relatif de l'analyse. «Le holisme ontologique de Rousseau, qui le pousse à réduire les prestiges de l'analyse, le conduit à reconnaître que le mixte est tout autre chose qu'une juxtaposition » (p. 154). Ce principe gouverne son épistémologie et son anthropologie.

Enfin, l'article de Bernardi, «Constitution et gouvernement mixte. Notes sur le livre III du Contrat social », montre comment Rousseau repose de manière originale, en s'appuyant sur la chimie, la question du gouvernement mixte, classique depuis Aristote. « Lequel vaut le mieux d'un gouvernement simple ou d'un gouvernement mixte ? (Le Contrat social, Paris, Garnier-Flammarion, 1966, III, 7, p. 117). Pour l'art politique véritable, attentif aux cas singuliers, il n'y a pas de gouvernement absolument meilleur, mais seulement des gouvernements relativement bons, dont il y a autant de formes que de cas possibles. La notion de gouvernement mixte permet de penser la multiplicité des combinaisons politiques possibles. Si Aristote part du simple pour penser, par composition, le mixte en politique, Rousseau part du gouvernement mixte pour remonter, par réduction, au gouvernement simple (démocratie, aristocratie, monarchie). Le gouvernement est un corps politique, dont les membres sont les magistrats. Plus ceux-ci sont nombreux, plus la force du gouvernement se dilue, plus le risque d'anarchie (confiscation de l'universel au profit du particulier) augmente. Aussi, le rapport des magistrats au gouvernement doit être inverse de celui des sujets au souverain. «Plus l'État s'agrandit, plus le gouvernement doit se resserrer, tellement que le nombre de chefs diminue en raison de l'augmentation du peuple » (Le Contrat social, op. cit., III, 2, p. 104). Rousseau rejette la typologie des formes simples de gouvernements au profit d'une évaluation des formes mixtes variant de façon continue. « Il y a un point où chaque forme de gouvernement se confond avec la suivante », le gouvernement étant «susceptible d'autant de formes diverses que l'État a de citoyens » (Le Contrat social, op. cit., III, 3, p. 106). La notion de gouvernement mixte permet ainsi de dissoudre la question du meilleur gouvernement en montrant qu' « elle a autant de bonnes solutions qu'il y a de combinaisons possibles dans les positions absolues et relatives des peuples » ( $L e$ Contrat social, op. cit., III, 9, p. 123). Rousseau redéfinit la notion de gouvernement simple en la situant dans un cadre analytique au lieu du cadre synthétique dans lequel elle s'était formée. «La chimie, parce que sa démarche est fondamentalement analytique, exige de nous que nous partions du divers et du multiple qui nous est donné, donnant à l'élémentaire le statut de résultat possible, au lieu de celui de présupposé et de principe » (Bernardi, p. 178). Cet examen comparé du Contrat social et des Institutions chymiques montre que Rousseau, en politique et en chimie, substitue à la démarche synthétique une approche analytique de l'élémentaire. La politique est l'art de produire, par une union serrée, un être moral doté de qualités propres, distinctes de celles des êtres particuliers qui le forment, de même que les composés chimiques ont des propriétés qu'ils ne tiennent d'aucun des éléments qui les composent. La politique, par analogie avec la chimie, est l'art de « changer 
pour ainsi dire la nature humaine, de transformer chaque individu, qui par lui-même est un tout parfait et solitaire, en partie d'un plus grand tout, dont cet individu reçoive en quelque sorte sa vie et son être »(Le Contrat social, op. cit., II, 7, p. 77). La politique trouve dans la chimie son paradigme : l'art de produire des corps par mixtion. Pour Rousseau, la chimie serait donc un « outil décisif dans la formation de sa problématique politique propre et la constitution de son système conceptuel » (p. 194).

Mai LEQUAN

Jean D'AlEMBERT, Discours préliminaire des éditeurs de 1751 et articles de l'Encyclopédie introduits par la querelle avec le Journal de Trévoux. Textes établis et présentés par Martine Groult. Paris, Honoré Champion, 1999. 14 × 22, 296 p. (L'Âge des Lumières, 7).

Rendre accessibles les différents textes qui sous-tendent la philosophie de l'aventure encyclopédique est l'objectif clairement affiché de cette édition, qui se compose de trois parties s'emboîtant les unes dans les autres, avec une évolution globalement chronologique.

La première partie reprend les polémiques qui marquèrent les débuts de la publication de l'Encyclopédie. Le Prospectus de Diderot, publié en novembre 1750, est immédiatement réfuté par les Jésuites dans le Journal de Trévoux. Les trois articles qui accusent Diderot d'avoir copié Bacon sans l'avoir compris sont reproduits ici, après le Prospectus, et chacun peut désormais participer au débat. Il ne s'agit pas évidemment de «laver ces grands hommes de l'affront d'avoir été considérés comme de vulgaires copistes » (p. 10) mais de « cibler» les points théoriques de divergences. L'article « Art », grand moment diderotien, est également reproduit pour clore définitivement les malheureux Jésuites au pilori de l'histoire.

La deuxième partie marque l'intervention de d'Alembert. Le Discours préliminaire des éditeurs est reproduit pour la première fois en France dans une édition séparée. Il s'agit du texte paru le 28 juin 1751, alors que la version le plus souvent rééditée est celle de 1759, la comparaison entre les deux Discours permet de valoriser la première version qui, selon Martine Groult «comporte l'extrême avantage de mettre en évidence un écrit destiné à fonder un nouveau courant de pensée » (p. 11). Pour rendre le dossier plus complet, l'Avertissement de l'Encyclopédie a été conservé avec l'Explication détaillée du système des connaissances humaines et les Observations sur la division des sciences du chancelier Bacon, ces derniers textes étant de Diderot. Ce second ensemble se termine par les Avertissements aux éditions de 1753, 1759, 1763, 1767.

La troisième partie est entièrement consacrée à d'Alembert. Elle comporte « les plus beaux textes de d'Alembert dans l'Encyclopédie », selon une logique épistémologique, qui restitue les « leçons de logique et de méthodologie du grand mathématicien aux philosophes » (p. 12).

L'ensemble du dossier est d'un grand intérêt. L'historien cependant aurait souhaité plus d'insistance sur la contextualisation. À lire Groult, on a l'impression que le grand débat entre philosophes et Jésuites tournait autour de Bacon et se situait dans le ciel des Idées. La réalité fut moins glorieuse. Si les jésuites ouvrent la guerre, et si les premiers affrontements demeurent théoriques, après l'attentat de Damiens (1757) les arguments mis en avant par les philosophes changent de registres. D'Alembert ne l'ignorait pas et son ouvrage sur la « destruction » des Jésuites (évoqué dans la note 6 page 9) reprenait contre les Jésuites, les accusations de tyrannicide et autres inventées par les jansénistes. En 1759, dans une lettre à Voltaire (citée p. 9), d'Alembert se félicite que « le frère Berthier et ses complices n'osent paraître actuellement dans les rues de peur qu'on ne leur jette des oranges de Portugal à la 
tête »; il s'agit bien du tyrannicide mis en avant par Pombal, et repris par le journal janséniste des Nouvelles ecclésiastiques... La défense des Lumières emprunte parfois des chemins obscurs.

Monique COTTRET

Denis Diderot, Choix d'articles de l'Encyclopédie. Anthologie établie et présentée par Marie LECA-Tsiomis. Paris, Éditions du C.T.H.S., 2001. 11,3× 18,4, 631 p., bibliogr. (Format, 44).

Éditée à l'occasion du $250^{\mathrm{e}}$ anniversaire de la publication du tome I de l'Encyclopédie de Diderot et d'Alembert, cette anthologie présente une sélection parmi les nombreux articles dont Diderot est l'auteur. Ce choix est réalisé par Marie Leca-Tsiomis qui, avec son livre Écrire l'Encyclopédie : Diderot, de l'usage des dictionnaires à la grammaire philosophique (Oxford, Voltaire Foundation, 1999), s'est imposée comme une des meilleures spécialistes actuelles de l'Encyclopédie. Dans une substantielle préface, elle restitue tout d'abord l'aventure de l'Encyclopédie qui, à l'origine, ne devait être qu'une traduction augmentée d'un ouvrage anglais et qui, avec ses vingt-huit volumes, ses nombreux collaborateurs et le quart de siècle employé à sa publication, fut au total la plus grande entreprise éditoriale de l'époque. L'histoire de la publication elle-même, entrecoupée de polémiques et d'interdictions, est à elle seule un des plus beaux témoignages de ce que fut le siècle des Lumières en France.

Dans ce vaste champ, c'est sur la contribution de Diderot que Leca-Tsiomis choisit de mettre l'accent, ce qui représente encore un immense gisement, même à ne s'en tenir qu'aux articles dûment identifiés comme siens. Comme le dit Leca-Tsiomis : « La sélection faite ici, discutable comme toute sélection, repose sur deux principes directeurs : d'une part faire apparaître la réflexion et la pratique de Diderot éditeur et coordinateur de l'ensemble ; et, d'autre part, donner à lire des articles qu'il rédigea comme collaborateur lui-même, principalement choisis dans les trois principaux domaines auxquels il se consacra: la description des arts et des métiers, la philosophie et son histoire, et la grammaire » (p. 24). L'article «Encyclopédie » mérite en premier lieu l'attention car Diderot, à la lumière des cinq volumes déjà publiés, y tire les leçons de l'expérience acquise, répond à de multiples critiques et, surtout, énonce ce qu'on peut considérer comme le programme même de l'Encyclopédie, les difficultés qu'elle doit rencontrer, les ambitions qu'elle vise à réaliser. Leca-Tsiomis met en valeur ce qui a constitué une des principales innovations de l'entreprise encyclopédique : la description des arts et métiers. On sent bien à la lecture d'articles comme « Gaze » ou « Émail » l'admiration de Diderot pour ce savoir technique à l'œuvre et la volonté d'en rendre compte dans les termes les plus clairs possibles. Elle rend aussi pleinement justice au travail de Diderot lexicographe, la pratique de la définition s'accompagnant toujours chez Diderot d'une réflexion sur l'art et la manière de bien définir.

Les principales critiques qu'on a envie de faire à cette anthologie, comme toujours, portent sur l'absence d'articles qu'on aurait aimé voir cités. C'est la loi du genre, et LecaTsiomis cite une phrase de Diderot qui s'applique ici fort bien : « Les omissions sont le plus grand défaut d'un dictionnaire [...]. Rien ne chagrine tant un lecteur que de ne pas trouver le mot qu'il cherche » (p. 28). Ici, par exemple, le philosophe regrettera l'absence des articles « Autorité politique », «Spinosiste », ou encore «Vice» et «Volonté ». Mais une autre absence chagrinera le lecteur mis en appétit par la préface, qui signale par exemple de nombreux emprunts de Diderot : celle de notes de bas de page de l'éditrice, qui éclaireraient 
les allusions, préciseraient l'identité des auteurs cités par Diderot, etc. (les notes se limitant pour l'essentiel aux traductions des citations latines).

Cela ne retire rien au plaisir de se promener dans cette anthologie, d'y découvrir la variété des styles de Diderot et l'étendue de ses préoccupations, et d'y lire ou d'y relire les différents articles, les «petits» (comme «Besançon» ou « Haire ») étant souvent moins connus mais tout aussi réjouissants que les « grands ».

Colas Duflo

Martine Groult, dir., L'Encyclopédie ou la création des disciplines. Paris, CNRS Éditions, 2003. 16,8 × 23,8, 346 p., front., ill., bibliogr., index.

Au XVIII ${ }^{\mathrm{e}}$ siècle, qui place l'entendement humain au premier plan, les philosophes, organisateurs d'idées, se trouvent associés aux savants, assembleurs d'idées. C'est cette union de la science et de la philosophie qui sert de point de départ au présent ouvrage, dans la mesure où elle a généré un «Système figuré des connaissances humaines » qui montre les liens reliant les sciences entre elles. Les spécialistes de l'Encyclopédie, rassemblés à l'École normale supérieure de Lyon en juin 2001, pour le 250e anniversaire de l'Encyclopédie, à l'initiative de Martine Groult, se sont plus précisément interrogés sur la création des disciplines résultant de cette nouvelle conception du savoir. L'ouvrage est divisé en trois parties : la première est consacrée à la classification des sciences, la deuxième aux sciences des arts et de la nature, la troisième aux sciences de la langue et de la politique. Il comporte en outre une longue bibliographie internationale et de stimulantes notes de l'éditrice, constituées à partir des discussions échangées lors du colloque entre les intervenants. Le frontispice de l'Encyclopédie et le «Système figuré des connaissances humaines » qui ouvrent le volume n'ont pas qu'une valeur ornementale, comme le montre Groult dans sa préface, ils participent du projet scientifique même de l'Encyclopédie.

Certes les éditeurs de l'Encyclopédie n'ont pas créé les disciplines dont ils rendent compte, mais ils ont inventé une structure permettant de représenter chaque discipline par ses éléments et de la définir par leur liaison. Grâce à cette « mise en discipline », il devenait possible de créer de nouvelles disciplines en empruntant des éléments à des disciplines différentes. L'ordre est ainsi conçu par d'Alembert dans le «Discours préliminaire » de l'Encyclopédie comme prioritaire sur la matière et surtout la métaphysique traditionnelle, d'ordre ontologique, est supplantée par une nouvelle métaphysique, qu'on a appelée la « métaphysique des corps ».

Dans cette perspective, la classification des sciences revêtait une importance capitale. C'est donc sur cette question que s'ouvre le recueil d'articles réunis par Groult. Henri Durel montre que, si Bacon est considéré à juste titre comme le père de l'Encyclopédie, il est également le petit-fils d'une encyclopédie française ayant inspiré l'auteur anglais, Tableaux accomplis des arts et des sciences (1587) de Christophe de Sauvigny. Bacon a remodelé le tableau buissonnant de Sauvigny en créant un système à deux niveaux qui comporte à la base l'histoire et au sommet la philosophie. Ellen Ruth Moerman remonte aussi la chaîne des filiations en examinant les lectures d'Ephraïm Chambers, auteur de la Cyclopaedia, source anglaise de l'Encyclopédie. En particulier, les traductions scientifiques de Chambers ont nourri ses connaissances dans différents domaines : la perspective avec Jean Dubreuil, la chimie avec Hermann Boerhaave, l'architecture avec Sébastien Leclerc, ainsi que la physique ou l'astronomie avec l'Histoire de l'Académie royale des sciences. Il n'est pas 
surprenant dès lors que Michel Malherbe ait pu s'intéresser à l'Encyclopédie comme œuvre de mémoire, qui à la fois rassemble des connaissances éparses (tableau) et les classe selon la raison (système). Mariafranca Spallanzani s'interroge sur la place de la philosophie dans ce classement. Absente du « Système figuré des connaissances humaines » et du « Discours préliminaire », celle-ci apparaît dans les articles au nom des différentes doctrines qu'elle produit, selon une conception qui en fait l'histoire de l'esprit humain et de ses disputes. Comme le souligne Groult dans son article, l'évolution du « Système figuré des connaissances humaines » entre le Prospectus de 1750 et le «Discours préliminaire » de 1751 marque un tournant dans l'histoire de la philosophie en faisant naître la métaphysique du raisonnement humain : « Il restait au public qui lit à intégrer la métaphysique comme logique de l'entendement pour saisir des dépendances mutuelles et créer, grâce aux vides, de nouvelles disciplines » (p. 90). La voie était ainsi ouverte pour la création d'une science de l'homme, qui devait aboutir à l'anthropologie. Une autre discipline se trouvait aussi en germe, selon Encarnación Medina Arjona, dans le «Discours préliminaire », à savoir la philologie comparée, dont Lorenzo Hervás y Panduro est reconnu comme le père. La postérité de la classification des sciences telle qu'on la découvre dans l'Encyclopédie se retrouve enfin dans un manuscrit latin, Atlas literarius... Prospectus, rédigé à Weimar en 1785. Françoise Bléchet, qui le décrit avec reproductions à l'appui, nous apprend qu'il se présente à la fois comme une table analytique, un système de classification et surtout une tentative de bibliographie universelle.

Après cette vaste enquête sur la classification des sciences qui situe l'Encyclopédie dans une longue lignée, cet ouvrage collectif aborde une autre des innovations apportées par le dictionnaire de Diderot et d'Alembert, qui réside dans la place faite aux arts. Il n'existe pas à proprement parler d'esthétique dans l'Encyclopédie, rappelle Jacques Proust, mais un article « Beau » de Diderot, qui a puisé dans ses lectures, celle du père Yves-Marie André notamment, et dans son expérience des ateliers des réflexions permettant de se forger une conception personnelle. Parallèlement, mais de manière complémentaire, l'image prend dans l'Encyclopédie une place de plus en plus grande, que nous pouvons suivre avec Madeleine Pinault Sørensen : pour Diderot, l'image constitue un moyen de diffusion du savoir au même titre que les textes. Un exemple de cette heureuse union du texte et de l'image nous est donné par Marie-Joelle Louison-Lassablière, dans un article consacré à l'orchestique. Le XVIII ${ }^{\mathrm{e}}$ siècle connaissant la prolifération des œuvres sur la danse, les encyclopédistes ne pouvaient manquer de s'y intéresser : ils relèvent le défi de mettre la danse en dictionnaire.

Quant aux sciences de la nature, elles sont particulièrement développées dans l'Encyclopédie. En passant du système général de la connaissance humaine proposé par Bacon à une explication détaillée des connaissances humaines, écrit Éliane Martin-Haag, les encyclopédistes ont conféré une importance nouvelle à la chimie par rapport à la physico-mathématique. La conception diderotienne qui fonde l'institution des disciplines sur l'imagination géniale éclaire l'article «Chymie » de Gabriel-François Venel : la notion de génie autorise la chimie à se donner pour une discipline autonome, ayant comme la physico-mathématique son génie propre. Mais l'un des traits les plus originaux de l'entreprise de Diderot est le combat qu'il mène pour l'émancipation des arts mécaniques. Paolo Quintili décrit sa stratégie de défense des artisans et des ouvriers, qui consiste à exalter les mérites de leur intelligence en les assimilant aux artistes libéraux et contribue à fonder une véritable technologie. En considérant, enfin, à partir de l'article « Hygiène » et de ses renvois, l'histoire de l'hygiène et la théorie physiologique sur laquelle l'Encyclopédie fonde les préceptes pratiques qu'elle énonce, Daniel Teysseire fait ressortir l'élargissement de l'hygiène en discipline d'action sociale.

La troisième partie du volume concerne la langue et la politique. Partant de l'héritage de Chambers et de la tradition lexicographique française, Marie Leca-Tsiomis relève 
l'introduction de la langue usuelle dans l'Encyclopédie et voit dans cette entreprise de grammaire philosophique un des traits caractéristiques de l'ouvrage. Sylvain Auroux attribue aussi un rôle fondateur à l'Encyclopédie à l'égard de la grammaire générale. Pour la première fois, on dispose grâce aux encyclopédistes d'un abondant traité sur les matières grammaticales. Sylviane Léoni s'est penchée sur le cas de la logique dans l'Encyclopédie en notant que, si l'acception du terme est variable d'un article à l'autre, la nature apparaît au centre du concept. David Diop note ensuite que l'Encyclopédie juxtapose deux méthodes d'étude de la politique, à savoir l'art politique, traditionnellement destiné au prince, et la science ou philosophie politique. La recherche, selon les termes de d'Alembert, des causes premières de la politique constitue la caractéristique de cette dernière. À défaut d'être une science, la politique est dans l'Encyclopédie une discipline ouverte. Nicolas Veysman analyse l'histoire voltairienne des opinions à la fois comme antithèse et comme antichambre de l'histoire des progrès humains, en la comparant à la conception de d'Alembert, qui continue à voir l'opinion comme une antichambre de la vérité scientifique mais ne la considère plus comme son antithèse. Jean-Claude Beaune envisage, pour finir, l'Encyclopédie à la fois comme pensée des machines et comme mégamachine, en posant le problème de l'unité de l'ouvrage.

À l'issue de cette vaste enquête, la spécificité du dictionnaire encyclopédique de Diderot et d'Alembert par rapport à ses satellites apparaît clairement au lecteur. Comme nous y invite Groult dans sa préface, il suffit pour s'en convaincre de faire un essai de lecture. Si on ouvre l'Encyclopédie pour connaître le sens du mot « discipline », on va trouver à la lettre D la définition de «discipline » comme instruction et gouvernement, accompagnée de sousarticles qui en donnent les définitions ecclésiastique et militaire. On pourrait en conclure que l'Encyclopédie ne connaît pas le sens du mot « discipline » tel qu'il est employé dans le titre de notre ouvrage. Mais si on la lit en commençant par le «Discours préliminaire », on aboutit vite à l'article «Art », qui fonde l'entreprise sur la construction de règles tendant à un même but. On se trouve en fait devant la définition philosophique de la discipline donnée plus loin par Diderot : «[Le] point de réunion auquel on a rapporté les observations qu'on avait faites, pour en former un système de règles ou d'instruments, et de règles tendant à un même but ; [...] voilà ce que c'est que discipline en général » (Encyclopédie, $1^{\text {re }}$ éd., Paris, 1751, t. I, p. 713b). De cette ambitieuse conception, l'entreprise encyclopédique est le stimulant reflet et ce foisonnant ouvrage, qui fera date, en témoigne.

Sylviane ALBERTAN-COPPOLA

Haydn Mason, dir., Les Euvres complètes de Voltaire. 9 : 1732-1733. Oxford, Voltaire Foundation, 1999. $15 \times 23$, XXV-531 p., bibliogr., index, ill. (The Complete Works of Voltaire, 9).

L'année 1732-1733 est celle qui précède le scandale des Lettres philosophiques. Selon le maître d'œuvre, l'ensemble de la production voltairienne, à ce moment, peut être lue en fonction de cette échéance fondamentale. Avant Cirey, Voltaire médite déjà sur les bienfaits d'un séjour en Angleterre, ou pourquoi pas en Avignon, pour éviter de fréquenter à nouveau la Bastille. Zaïre remporte pourtant un grand succès à la Comédie-Française, mais son « Épître dédicatoire » n'obtient pas le privilège d'imprimer. En dédiant l'œuvre à un marchand anglais, Voltaire célèbre déjà les vertus du modèle anglais qui encourage les écrivains, tandis que la France n'est plus à la hauteur du siècle de Louis XIV. De quoi irriter effectivement les autorités. 
Face aux menaces du garde des Sceaux, Germain Louis de Chauvelin, Voltaire tremble pour les Lettres, qui paraissent pourtant en anglais (en août 1733), sous le titre de Letters concerning the English Nation, et connaissent un grand succès.

L'agitation autour de cette œuvre fondamentale, comme les querelles habituelles, ici avec Jean-Baptiste Rousseau, n'entament pas la frénétique activité de Voltaire. La préface rappelle qu'il termine alors Adélaïde Du Guesclin, rassemble les documents en vue du Siècle de Louis XIV, compose le livret de Samson, revoit Eriphyle... Il y a de quoi se perdre dans cette effervescence créatrice, et l'on comprend Voltaire constatant : « J'efface, j'ajoute, je barbouille. La tête me tourne » (p. XXv).

Mise en perspective et vision rétrospective éclairent les œuvres présentées, chacune avec une introduction qui la situe dans le corpus voltairien comme dans l'époque, une liste très utile des différentes éditions, et d'indispensables notes érudites. O. R. Taylor se consacre au Temple de l'amitié (p. 3-24) puis au Temple du goût (p. 27-256) ; Sylvain Menant met en lumière un petit «badinage », le terme est du roi de Prusse, La Mule du pape (p. 219-269) ; D. J. Fletcher s'intéresse à l'Épître sur la calomnie (p. 273-308); Pierre Rétat éclaire la Lettre à un premier commis (p. 311-322); Samuel S.B.Taylor livre une étude de la Vie de Molière (p. 325-463) ; Nicole Masson et Sylvain Menant ont annoté des Poésies diverses (p. 466-504). Une liste des ouvrages cités et un index terminent heureusement ce beau travail.

Monique CoTTReT

René TARIN, Diderot et la Révolution française. Controverses et polémique autour d'un philosophe. Préf. de Roland Desné. Paris, Honoré Champion, 2001. 16,5 × 24, 189 p., index (Les Dix-Huitièmes siècles, 55).

Avec ce nouvel ouvrage, René Tarin contribue à l'étude du rapport entre la pensée des Lumières et la Révolution, en choisissant d'examiner plus particulièrement l'écho suscité par l'œuvre de Diderot. Pour cela, il évoque les différents débats dans lesquels le nom de Diderot apparaît : les colonies et l'esclavage (prologue et chap. I), les questions des liens de la morale et de la religion (chap. II), de l'extension des droits du peuple (chap. III), du rôle et de l'usage politique de l'art (chap. IV), la question de la propriété légitime (chap. v) et enfin le débat virulent à propos des couvents (chap. vI). Chaque chapitre est suivi d'une bibliographie spécifique récente, et l'ouvrage est enrichi d'illustrations. Trois index (des noms, des œuvres et des périodiques) aident encore à la lecture. L'auteur démontre que le nom de Diderot sert à identifier des positions plus politiques que philosophiques. L'édition progressive des œuvres contraint certes les différents protagonistes à diversifier leur discours sur Diderot. C'est pourtant, d'après Tarin, un « combat perdu d'avance » (p. 162) que les défenseurs de Diderot livrent contre la caricature que la période révolutionnaire lègue au XIX ${ }^{\mathrm{e}}$ siècle.

L'ouvrage suit la réception des œuvres en fonction des événements politiques de la France révolutionnaire. Les chapitres consacrés à l'Histoire des deux Indes sont naturellement articulés à la question coloniale. Ils mesurent l'importance du nom de Diderot dans ce débat, en montrant que l'Histoire des deux Indes est à de nombreux points de vue le texte de référence lors des débats parlementaires. Tarin note l'évolution des positions de Diderot lui-même, puis il cherche à établir quels sont les fragments qui sont utilisés dans le débat révolutionnaire, à quelles fins, et par qui, à l'aide de sources historiques primaires telles que les discours parlementaires ou les journaux. L'auteur interprète ainsi ce qu'il nomme le « reniement de Raynal » par des considérations de politique extérieure. 
Le chapitre consacré à « Robespierre et le matérialisme de l'Encyclopédie » procède selon la même démarche. Tarin démontre que l'opposition de Robespierre à ce que « l'Incorruptible » nomme «l'opinion du matérialisme » et de « l'athéisme », que ce dernier attribue hâtivement à l'ensemble des encyclopédistes, est en fait un « choix idéologique » dicté par « les exigences de l'histoire et les impératifs de l'heure» (p. 58). La reprise des termes mêmes de « l'analyse » de Robespierre mériterait néanmoins un examen préalable : peut-on parler du « compromis » de la « secte des encyclopédistes » avec les « figures de proue de l'aristocratie nobiliaire et de l'élite sociale» (p. 53), et si nul ne conteste le «matérialisme exprimé par certains articles de l'Encyclopédie », peut-on dire uniment que «l'Encyclopédie de Diderot traduit cette nouvelle conception de l'homme » (p. 45), ou que «l'élaboration d'une "religion naturelle" ouvrait la voie vers le matérialisme» (p. 50) ? Le chapitre « Théorie politique et droits du peuple» réitère et complète la démonstration en matière politique. Il montre comment Robespierre utilise les articles de l'Encyclopédie qui lui semblent défendre le despotisme éclairé, pour mieux assimiler les héritiers revendiqués des Lumières à la coalition de monarchies étrangères contre la France.

Dans le domaine esthétique, Tarin soutient l'idée d'une correspondance entre les thèses de Diderot et la politique culturelle de la Révolution, à propos de laquelle il nous a déjà offert Le Théâtre de la Constituante ou l'école du peuple (1998). Le peu de succès des journalistes de la Décade, qui insistent sur cette dimension de l'œuvre diderotienne pour la réhabiliter, devient alors problématique. L'auteur l'explique au chapitre suivant par l'association de Diderot à Babeuf, via l'attribution à Diderot du Code de la nature, revendiqué par Babeuf comme sa principale source d'inspiration. À la suite du procès des Égaux, JeanFrançois de La Harpe et Augustin Barruel vont jusqu'à faire de Diderot le « destructeur de la morale et de l'ordre social par le moyen du massacre général »... Jean-André Naigeon précipite alors la sortie des Euvres de Diderot, espérant rééquilibrer cette lecture de Diderot centrée sur l'Histoire des deux Indes et le Code de la nature, mais en vain.

L'auteur montre que cette publicité tapageuse faite à l'œuvre de Diderot, complétée de quelques autres scandales (à propos des Éleuthéromanes et de La Religieuse pendant l'été 1796), n'entame cependant pas le désintérêt dont souffre l'œuvre du philosophe. Grâce au tableau dressé par Tarin, on comprend mieux la perplexité des premiers lecteurs de Jacques le Fataliste, peu préparés à découvrir un écrivain si peu dogmatique et si peu respectueux des genres littéraires. Seuls des membres de la $2^{\mathrm{e}}$ classe de l'Institut tentent de rendre justice à Diderot : Tarin suggère, en épilogue, une filiation entre Diderot et ces héritiers lointains, via La Décade, dont fait preuve un Éloge philosophique de Denis Diderot par Eusèbe Salverte. Cette très stimulante hypothèse correspond à une véritable interrogation concernant la postérité philosophique de Diderot, et on aimerait disposer d'une analyse allant dans le détail des arguments à ce propos - mais ce serait là un autre ouvrage.

Le livre de Tarin fait donc à nouveau apparaître combien la postérité immédiate de Diderot fit de lui le champion de causes opposées, pour avoir lu l'œuvre de Diderot non comme un ensemble, même non systématique, mais comme la somme de défenses locales de thèses disparates, voire contradictoires. 
Marc Schalenberg, Humboldt auf Reisen? Die Rezeption des «deutschen Universitätsmodells » in den französischen und britischen Reformdiskursen (1810-1870). Bâle, Schwabe \& Co. Verlag, 2002. $23 \times 15,520$ p., bibliogr., index (Veröffentlichungen der Gesellschaft für Universitäts- und Wissenschaftsgeschichte, 4).

Fruit de la thèse (soutenue en mai 1999) d'un jeune historien allemand, lui-même héritier de la tradition qu'il prend pour objet d'étude, cette monographie, sans jamais céder à l'effet de mode ou à l'opportunisme conjoncturel, offre l'intérêt non seulement d'un travail de recherche sérieux mais aussi l'attrait d'un bilan, à un moment où l'Europe universitaire s'interroge sur les manières de construire l'avenir de la science et de l'enseignement. Le $\mathrm{XIX}^{\mathrm{e}}$ siècle, qui se trouve au centre de la démarche, est à la fois le creuset et la source, en lequel culminent toutes les aspirations d'une nation avide d'identité et de rayonnement. Non seulement tournée vers l'édification d'une cohérence institutionnelle interne, l'Allemagne attirait également les regards de ses voisins, qui trouvèrent en elle un lieu d'inspiration et un modèle. Tandis que l'intérêt pour la littérature et la pensée (Kant, Hegel, Goethe, Schiller) dépassa le cadre de toute structure institutionnelle, l'attention pour le système universitaire apparaît curieuse et critique à la fois.

Le siècle qui s'ouvre avec les principes édictés par Humboldt est, pour l'Allemagne, celui de l'affirmation volontariste de son identité «culturelle». La science et la culture à l'allemande tracent leur voie à l'intersection de postulats théoriques et de réalisations pratiques. L'accent mis sur la haute mission de l'enseignement universitaire, clairement séparé de l'enseignement secondaire, donne au système allemand le caractère d'une structure semper reformanda, produit et productrice à la fois de ses propres idéaux. Tout au long du siècle, et au delà, la pratique n'est jamais séparable de la théorie, avec laquelle dans bien des cas elle se confond. L'étude de Schalenberg se situe dans la perspective d'une histoire des idées « contextualisante », revendiquant un triple modèle épistémologique - réception et perception, portée de l'imitation, répercussions institutionnelles du modèle - et faisant appel à des critères aussi divers que la professionnalisation, la modernisation, l'importance du milieu, la logique de système, la construction d'une téléologie idéaltypique. Se plaçant pour ainsi dire au centre d'un nouvel idéal, l'Allemagne devient le parangon de la production scientifique universitaire, à côté d'une France tout différemment engagée dans la voie pragmatique de la « formation » et de la régénération nationale par l'éducation, et d'une Angleterre creusant la singularité de son sillon. Pour corriger le topos de la docte Allemagne prisonnière parfois de ses propres illusions, l'auteur de cette étude met à contribution divers types de sources à l'intérieur des limites chronologiques significatives d'un siècle marquant (1810: fondation de l'Université de Berlin ; 1870 : « année terrible », selon la formule de Victor Hugo) : textes officiels, rapports, circulaires, témoignages autobiographiques, etc.

L'analyse des principales caractéristiques de l'Université allemande fait l'objet du premier chapitre, dans lequel Schalenberg tente de cerner moins l'essence du système que l'image issue de sa perception externe. Les figures de Heyne, Wolf, Schiller, Fichte, Schleiermacher et Humboldt traversent l'évocation de la genèse d'une institution née de la volonté politique de la Prusse de travailler moins à son renouveau spirituel qu'à l'édification d'une identité nationale entièrement nouvelle, fondée sur l'unification de l'Allemagne et transcendant le territorialisme politico-religieux. Les intentions programmatiques des fondateurs reflètent l'orientation antifrançaise de l'entreprise : complémentarité voire unité de la science et de l'enseignement, liberté de la recherche, de l'enseignement et des études, caractère directif et directeur d'un état culturel, priorité de l'orientation scientifique. L'impératif, presque catégorique, de la recherche à tout prix, qui devient l'objectif des universités allemandes tout au long du XIX ${ }^{\mathrm{e}}$ siècle, est promu aussi bien à l'occasion d'expositions 
universelles que par des déclarations d'intention. L'idée d'université est la base et la clé de cette entreprise idéologique de construction d'une nation. Au titre de symbole, Humboldt en est l'incarnation. Le caractère irréprochablement objectif de l'analyse ouvre la voie aux volets suivants, où l'auteur décrit et apprécie les répercussions du modèle allemand sur l'évolution des systèmes respectivement français et britannique.

Les éléments fournis dans le chapitre relatif aux écoles supérieures françaises, s'ils instruiront sans doute plus utilement les lecteurs allemands que français, n'en restent pas moins précieux pour ces derniers du point de vue de l'économie générale du projet. Les principaux jalons de l'histoire institutionnelle de l'École normale supérieure, de l'École pratique des hautes études, de l'École polytechnique, des écoles spéciales, des collèges royaux, du Muséum national d'histoire naturelle, de l'Observatoire, du Conservatoire des arts et métiers, de l'École des chartes, du Collège de France, de l'Institut de France, et le rôle des missions scientifiques et littéraires sont évoqués ici dans l'optique d'une appréciation synthétique du rayonnement allemand et des mérites comparés de deux systèmes dont la géographie et l'histoire politique accentuent tout particulièrement au XIX siècle le voisinage. Le contexte historique napoléonien, le rayonnement personnel de figures littéraires telles que Madame de Staël, l'importance du milieu journalistique, l'activisme réformateur de la génération de 1830, le rôle missionnaire des Allemands séjournant ou travaillant en France, au nombre desquels un nombre significatif de philologues (L. Bornes, E. Gans, S. Munk, J. Oppert, J. Derenbourg, H. Weil, J. Mohl, K.-B. Hase, F. Dübner, L. Bamberger, K. Hillebrand), la détermination des passeurs de frontières dans le domaine de l'enseignement scientifique aussi bien que littéraire (L. Pasteur, E. Renan, H. Taine, G. Monod, G. Pouchet, E. Alglave), l'autorité des réformateurs (H. Fortoul, V. Duruy), l'importance des salons (Marie comtesse d'Agoult), la diffusion des périodiques sont autant de facteurs, divers et complémentaires, d'une science et d'une formation à la française, d'où l'auteur a l'intelligence de ne pas écarter la province (Strasbourg, Toulouse notamment) et la part prise par les sociétés savantes.

Le système des écoles supérieures britanniques paraît en comparaison à la fois plus simple et plus complexe. La tension dialectique entre l'autorité du modèle externe (R. L. Edgeworth, H. Crabb Robinson, T. Beddoes) et l'attachement viscéral à des principes internes éclaire l'engouement des uns (Noetics et Tractarians : E. Copleston, J. Davison, E. Hawkins, T. Arnold) et la tergiversation - pleine de réticence, «nolumus germanizari» (nous refusons d'être germanisés) - des autres (H. L. Mansel, G. Swayne, J. W. Burgon). Les rouages du système anglais, défini comme l'envers du miroir allemand, sont détaillés selon les mêmes principes d'exhaustivité et de distanciation critique que les composantes des institutions françaises : fonctions respectives de l'université (University of Oxford) et des «colleges », poids des bibliothèques (Bodleian Library), rôle des institutions scientifiques périphériques (Taylorian Institution, University Museum, Oxford Union, Royal Commission, Endowment of research movement, Jowett \& Co.), des structures parallèles comme les clubs et les revues (le cas de John Stuart Mill). Autrement qu'en France, les Allemands surent insuffler à la science et à sa transmission une direction nouvelle sans jamais inquiéter toutefois l'enracinement dans une tradition singulière (F. M. Müller, A. H. Niemeyer, V. A. Huber, L. Wiese, J. Liebig, C. C. J. von Bunsen). De même que l'auteur regarde en dehors de Paris pour la France, de même les cas emblématiques de Londres, Oxford et Cambridge se trouvent complétés par la prise en compte des universités écossaises ou irlandaises.

La conclusion, qui forme à elle seule une partie, apporte, avec toutes les nuances souhaitables, des réponses ou esquisses de réponses aux questions qui commandent cette enquête, notamment : a-t-il existé un modèle universitaire prusso-allemand bien défini qui aurait rayonné en Europe et s'y serait répandu au cours du XIX e siècle ? Par-delà l'attrait du nom de Humboldt en tant qu'étiquette ou slogan personnalisant un idéal de grandeur et de 
conquête intellectuelle, l'Angleterre et la France ont puisé surtout dans le système allemand une légitimation complémentaire de leur tradition propre, à partir du modèle de vie et de pensée incarné par Humboldt et postulant le dépassement de l'humanisme traditionnel et l'édification d'un nouvel humanisme propre à l'homme moderne. Vue du dehors, l'université allemande offrait l'image parfaitement léchée d'une entité idéale, où maîtres et disciples, enseignants et étudiants travaillaient en harmonie, et où se trouvaient mis en avant le rôle de l'État, la liberté comme valeur suprême, la volonté d'innovation, le désintéressement, la science pure et l'importance de la recherche fondamentale. L'université allemande fonctionna un peu au dehors comme une image d'Épinal et un idéal borné par sa perfection théorique. Ce que les uns et les autres surent en retenir la définit mieux peut-être que ce qu'elle réalisa elle-même en son propre sein. L'imiter, toujours partiellement, revenait en définitive à en corriger les excès. Entre référence positive et référence négative, elle valut (et continue de valoir) surtout par l'expérience qu'elle incarne, celle d'une canalisation dirigiste et institutionnelle de la production intellectuelle. Les remarques conclusives de Schalenberg ont l'élégance d'ouvrir la voie à une appréciation juste et contradictoire des arguments que son enquête fournit.

Une bibliographie impressionnante en volume et en qualité couronne l'ouvrage de la plus belle façon. La somme analytique et documentaire que constitue ce livre lui vaudra de retenir durablement l'attention de lecteurs qu'il faut souhaiter nombreux.

Pascale HuMmel 